\title{
An alternative theoretical approach for the derivation of analytic and numerical solutions to thermal Marangoni flows
}

\author{
Marcello Lappa ${ }^{1}$ \\ ${ }^{1}$ Department of Mechanical and Aerospace Engineering, University of Strathclyde, James Weir \\ Building, 75 Montrose Street, Glasgow, G1 1XJ, UK - email: marcello.lappa@strath.ac.uk
}

\begin{abstract}
The primary objective of this short work is the identification of alternate routes for the determination of exact and numerical solutions of the Navier-Stokes equations in the specific case of surface-tension driven thermal convection. We aim to introduce a theoretical approach in which the typical kinematic boundary conditions required at the free surface by this kind of flows can be replaced by a homogeneous Neumann condition. More precisely, the novelty of the present framework lies in the adoption of a class of 'continuous' distribution functions by which no discontinuities or abrupt variations are introduced in the model. The rationale for such a line of inquiry can be found 1) in the potential to overcome the typical bottlenecks created by the need to account for a shear stress balance at the free surface in the context of analytic models for viscoelastic and other non-Newtonian fluids and/or 2) in the express intention to support existing numerical (commercial or open-source) tools where the possibility to impose non-homogeneous Neumann boundary conditions is not an option. Both analytic solutions and (two-dimensional and three-dimensional) numerical "experiments" (concerned with the application of the proposed strategy to thermocapillary and Marangoni-Bénard flows) are presented. The implications of the proposed approach in terms of the well-known existence and uniqueness problem for the NavierStokes equations are also discussed to a certain extent, indicating possible directions of future research and extension.
\end{abstract}

Key words: Marangoni flow, analytic solutions, two-dimensional and three-dimensional numerical results.

\section{Introduction}

Gravitational and surface-tension driven convection in fluid-filled enclosures has received considerable attention over the past several years due to its extensive background applications in engineering design of advanced technology. These applications (of practical or prototypical natures, too many to be cited here) span such diverse fields as electronic industry, cooling plants, coating processes, organic and inorganic crystal growth, etc (Melnikov et al. [1], Melnikov and Shevtsova [2], Li et al. [3], Xun et al. [4], Henry et al. [5]).

Leaving aside for a while applicative aspects, such subjects have also attracted significant academic interest. Indeed, the instabilities of these flows and related hierarchy of bifurcations do exert an appeal to researchers and scientists because of the variety of patterns and related spatio-temporal evolution. Such features are often aesthetically pleasing and "philosophically" challenging (because of their implications in the development of a general theory for non-linear systems), which make 
them irresistible to theoretical physicists. Much interest also comes from the well-known inherent difficulties in elaborating a general theory able to predict such characteristics "a priori”.

One way to mitigate this drawback is to introduce a preliminary classification of the possible regimes and related solutions on the basis of the thermal and mechanical boundary conditions affecting the considered problem. A mathematical formulation of all such conditions leads to the socalled initial-boundary value problem (IBVP) where the governing balance equations for mass, momentum and energy have to be solved together with the related initial and boundary conditions. In turn, this requires implicitly the adoption of a given solution strategy, be it analytical, approximate or "numerical".

By analytical approach we mean one of the standard methods for classical partial differential equations (the obvious outcome of such a process being an algebraic expression relating the dependent variables to the independent variables, see, e.g., Ostroumov [6], Birikh [7,8], Gershuni and Zhukhovitskii [9], Belghazi et al. [10], Lappa [11], Lappa and Ferialdi [12]). An approximate method results when the governing boundary value problem is solved using a series-expansionbased technique (see, e.g., Jane [13], Jebari et al. [14], Al-Saif et al. [15]) or a transformation is used, based on the introduction of a similarity variable, by which the original partial differential equations are replaced by a set of coupled nonlinear ordinary differential equations (e.g., Makinde and Olanrewaju [16]). Finally, by numerical solution here we refer to the discrete set of nodal values that is obtained when the IBVP is integrated numerically (by discretizing the equations over a computational grid, see, e.g., [17-33]).

Unlike the analytical approach, however, none of these approximate numerical methods is able to yield a closed expression for the velocity in the flow in terms of driving forces involved and conditions at the system boundary.

This is the main reason for which analytic solutions of Marangoni convection have enjoyed a widespread use in the literature as a paradigm model for establishing (in general) a theoretical foundation to the field of surface-tension driven flows and (in particular) for explaining some of the typical manifestations of this kind of convection in practical situations.

These flows are known to undergo a variety of instabilities when the characteristic parameter (the so-called Marangoni number) exceeds one or more thresholds. Analytic solutions have allowed gaining outstanding insights into such behaviors due to their natural "ability" to be used as initial conditions (the so-called "basic flow") for straightforward application of linear-stability-analysis (LSA) techniques.

By contrast, when such a flow has to be determined numerically, the typical protocols of LSA require the solution of an eigenvalue problem of very high order (in practice, the order of this problem is equal to the amount of scalar unknowns used to represent the solution numerically, namely, a number given by the product of the number of unknown functions and the number of discretization elements, i.e. grid points, control volumes or finite elements effectively used).

Given such premises, it is really difficult to imagine how our understanding of these flows and their instabilities could have flourished without the availability of analytic solutions. Indeed, studies of 
buoyancy and Marangoni flow have historically progressed through the introduction of a precise hierarchy of models with increasing complexity where new knowledge was iteratively produced on the basis of the intuition developed on the basis of earlier simpler models. As an example, this approach proved extremely useful in the development of a general theory of flow bifurcation and turbulence (Lin [34], Rosenbluth and Simon [35], Drazin and Howard [36], Hart [37,38], Gill [39], Smith and Davis [40], Laure and Roux [41], Gershuni et al. [42], Parmentier et al. [43], Priede et al. [44], Priede and Gerbeth [45], etc.).

Before being impressed by the power of this idea, however, we have to warn that the derivation of analytical solutions to the Navier-Stokes equations is an extremely hard task. Indeed, only a very limited set of exact solutions is known (most of such solutions were originally published in a number of Soviet-Union journals, hardly accessible in the western world, and for this reason many of them have been ignored for a long time, see, e.g., Ostroumov [6]).

Technically speaking, in general, it is possible to find or "build" analytical solutions when the convective terms in the governing equations, namely the main sources of non-linearity, vanish naturally. Towards this end, some specific simplifications can be considered such as a reduction of the number of space dimensions involved or the "removal" of physical boundaries along certain directions (ideally assumed to be located at an infinite distance where they are not able to influence the "core" flow).

Despite these assumptions, however, significant drawbacks opposing to the straightforward determination of results in analytical form persist. For Marangoni flow an additional bottleneck is represented by the need to satisfy a non-homogeneous kinematic boundary condition at the free interface, which should be regarded as the main motivation for which further developments in this field have been relatively limited. This is especially true for the case of non-Newtonian fluids, for which such a boundary condition (a shear stress balance) becomes particularly complex and cumbersome because of the presence of other (e.g., viscoelastic) stresses in the fluid (in addition to the standard Newtonian and thermocapillary ones). Among other things, the non-homogeneous nature of this boundary condition has acted in many circumstances as a kind of "barrier" limiting the utilization of widespread commercial or open-source CFD tools (such as Open-Foam). Though many of such computational tools are equipped with a variety of functions and models (including the possibility to simulate viscoelastic fluids), often they lack the possibility to implement kinematic boundary conditions such as those that would be required to simulate thermal Marangoni convection.

Motivated by this observational tide, the main aim of the present analysis is to propose widening the range of methodologies to be potentially used to treat this kind of flows. In particular, we further develop and expand the approach originally introduced by Tiwari and Nishino [46] about the possibility to turn the stress-balance Marangoni condition at the liquid-gas interface into an equivalent condition or source term to be added directly to the momentum equation as it was a force of buoyancy nature. 


\section{Governing equations}

For simplicity, we build our framework on the assumption that the flow is laminar, steady and incompressible with constant properties.

\subsection{Nondimensional form}

In order to derive analytic solutions in the most general form, obviously, the governing equations have to be put in a non-dimensional shape. Here we consider the typical (most general) choice of characteristic reference quantities for thermal convection $([47,48])$; namely, we scale lengths, velocity, time and pressure by $d, V_{\alpha}=\alpha / d, d^{2} / \alpha$ and $\rho \alpha^{2} / d^{2}$, respectively, where $d$ is a reference distance, $\alpha$ is the fluid thermal diffusivity (and $\rho$ its density), and $V_{\alpha}$ is the energy diffusion velocity. Moreover, we subtract a reference value $\mathrm{T}_{\mathrm{o}}$ to the temperature, and scale it by a reference temperature difference $\Delta \mathrm{T}$. This approach leads to cast the mass, momentum and energy balance equations as:

$\underline{\nabla} \cdot \underline{\mathrm{V}}=0$

$\frac{\partial \underline{V}}{\partial t}+\underline{\nabla} \cdot[\underline{V} \underline{V}]+\underline{\nabla} p=\operatorname{Pr} \nabla^{2} \underline{V}+\underline{F}_{b}$

$\frac{\partial T}{\partial t}+\underline{\nabla} \cdot[\underline{V} T]=\nabla^{2} T$

where, $\underline{\mathrm{V}}, \mathrm{p}$ and $\mathrm{T}$ are the non-dimensional velocity, pressure and temperature respectively (the socalled "primitive variables"), $\underline{F}_{b}$ is a generic body force (e.g., buoyancy), and Pr is the Prandtl number $(\operatorname{Pr}=v / \alpha$ and $v$ is the constant kinematic viscosity $v=\mu / \rho)$.

To put the work in perspective, in the next two sections we illustrate the differences between classical approaches implemented in the past and the present one.

\subsection{Classical Analytic Solutions for Marangoni Flows}

As the resulting framework is not restricted to a specific geometry or model, without loss of generality we concentrate on the classical case of parallel flows $[7,40]$.

As shown in Fig. 1, initially we consider a laterally unbounded horizontal layer of liquid delimited from below by a solid wall and from above by a liquid-gas interface. The horizontal boundaries are assumed to be located at $y=-1 / 2$ and $1 / 2$, respectively. Moreover, there are no velocity components along $\mathrm{y}$ and $\mathrm{z}(\mathrm{v}=\mathrm{w}=0)$ while the component along $\mathrm{x}$ depends on the vertical coordinate $\mathrm{y}$ only, i.e. $\mathrm{u}=\mathrm{u}(\mathrm{y})$. The temperature undergoes a linear increase along the horizontal coordinate $\mathrm{x}$ (with $\mathrm{a}$ constant rate of increase $\gamma$ ), and as a result of fluid being transported with velocity $u(y)$, it also displays a dependence on the vertical coordinate (in other words, these analytic solutions are 
essentially two-dimensional). Moreover, we define the Rayleigh and Marangoni numbers as $\mathrm{Ra}=\mathrm{GrPr}=\mathrm{g} \beta_{\mathrm{T}} \gamma \mathrm{d}^{4} / \nu \alpha$ and $\mathrm{Ma}=\mathrm{RePr}=\sigma_{\mathrm{T}} \gamma \mathrm{d}^{2} / \mu \alpha$, respectively (where $\mathrm{d}$ is the distance between the boundaries, $\beta_{\mathrm{T}}$ is the thermal expansion coefficient, $\sigma_{\mathrm{T}}$ is the surface tension derivative with respect to temperature, Gr and Re are the Grashof and Reynolds numbers, respectively).

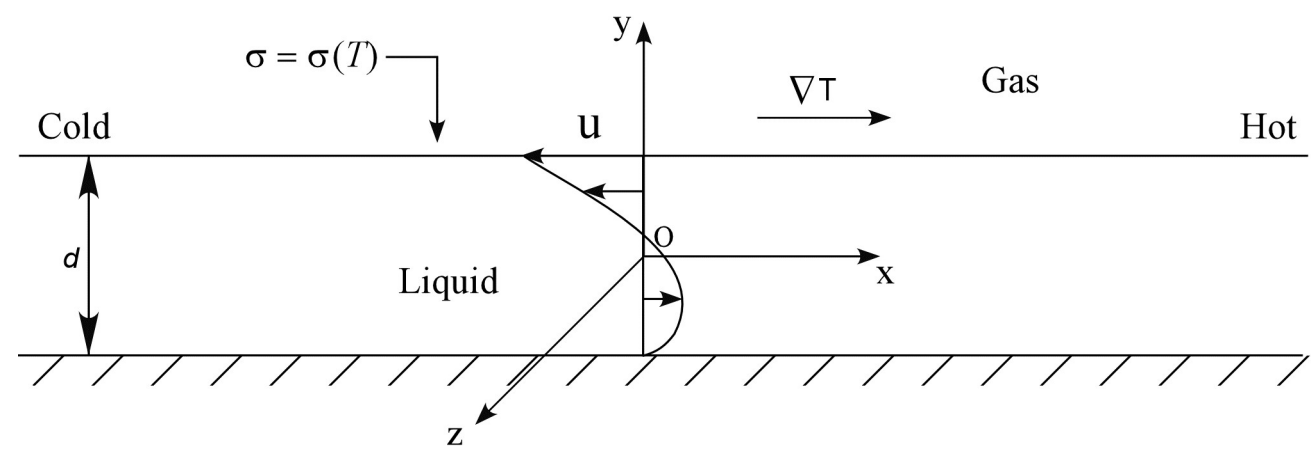

Fig. 1: Sketch of layer of infinite extent subject to horizontal heating.

In the presence of a free liquid/gas interface, obviously, equations (1)-(3) must be considered together with the so-called Marangoni boundary condition. If viscous stresses in the gas are neglected (in general such assumption is reliable because the dynamic viscosity of the gas surrounding the free liquid surface is much smaller than the viscosity of the considered liquid) such condition reads:

$\frac{\partial \underline{V}_{S}}{\partial n}=-M a \underline{\nabla}_{S} T$

where $\underline{\mathrm{n}}$ is the direction perpendicular to the free interface (planar in our case), $\underline{\nabla}_{S}$ the derivative tangential to the interface (the x direction) and $\underline{V}_{\mathrm{s}}$ the surface velocity vector.

This condition enforces a flow through the variation of the surface tension. Typically, this variation leads fluid located in proximity to the gas-liquid interface to move from regions of higher temperature towards relatively cold areas. The viscosity then transfers this motion to the underlying fluid, thereby turning an initial surface effect into bulk fluid motion.

For the case considered here (steady flow with $\mathrm{u}=\mathrm{u}(\mathrm{y}), \mathrm{v}=\mathrm{w}=0$ and perfectly flat and horizontal interface, as shown in Fig. 1) eqs. (1)-(3) reduce to :

$$
\begin{aligned}
& \frac{\partial p}{\partial x}=\operatorname{Pr} \frac{\partial^{2} u}{\partial y^{2}} \\
& \frac{\partial p}{\partial y}=\operatorname{Pr} R a T \\
& u \frac{\partial T}{\partial x}=\frac{\partial^{2} T}{\partial x^{2}}+\frac{\partial^{2} T}{\partial y^{2}}
\end{aligned}
$$

while eq. (4) can be rewritten as: 
$\frac{\partial u}{\partial y}=-M a \frac{\partial T}{\partial x}$

representing the aforementioned non-homogeneous Neumann boundary condition.

Assuming solutions in the general form (with $\mathrm{Ma}=0$ or $\mathrm{Ra}=0$ for pure thermogravitational or pure thermocapillary flows, respectively):

$\underline{V}=\left[\begin{array}{c}u \\ v \\ w\end{array}\right]=\left[\begin{array}{c}\operatorname{Ra} g_{1}(y)+M a g_{2}(y) \\ 0 \\ 0\end{array}\right]$

$T=x+R a f_{1}(y)+M a f_{2}(y)$

$f_{1}, f_{2}, \mathrm{~g}_{1}$ and $\mathrm{g}_{2}$ can be "built" as polynomial expressions, provided the related (polynomial) coefficients satisfy the alternate set of equations that can be obtained by substituting eqs. (9) and (10) into eq. (7) and into the equation resulting from cross-differentiation of eqs. (5) and (6) (i.e. $\left.\frac{d^{3} u}{d y^{3}}=R a \frac{\partial T}{\partial x}\right)$.

For the case of interest here, i.e. pure Marangoni flow ( $\mathrm{Ra}=0)$, such system of equations reduces to:

$g_{2}=\frac{d^{2} f_{2}}{d y^{2}}$

$\frac{d^{3} g_{2}}{d y^{3}}=0$

to be supplemented with the proper boundary conditions:

Kinematic conditions:

solid boundary: $\mathrm{u}=0 \rightarrow \mathrm{g}_{2}(\mathrm{y})=0$

free surface: eq. (8) $\rightarrow \frac{d g_{2}}{d y}=-1$

Thermal conditions:

Adiabatic boundary: $\frac{\partial T}{\partial y}=0 \rightarrow \frac{d f_{2}}{d y}=0$

Conducting boundary: $\mathrm{T}=\mathrm{x} \rightarrow f_{2}=0$ 
An additional equation constraining the "shape" of the final polynomial expressions for $g_{2}$ can be obtained by imposing that the net flux of fluid at any cross section of the slot must be zero to satisfy mass conservation, i.e.:

$$
\int_{-1 / 2}^{1 / 2} u d y=0 \rightarrow \int_{-1 / 2}^{1 / 2} g_{2} d y=0
$$

Known exact solutions ([7]) for such a system of equations (eqs. (11)-(17)) can be cast in compact form as:

$$
u=-\frac{M a}{4}\left(3 y^{2}+y-\frac{1}{4}\right)
$$

with:

$$
T=x-\frac{M a}{48}\left(3 y^{4}+2 y^{3}-\frac{3}{2} y^{2}-\frac{3}{2} y-\frac{5}{16}\right)
$$

for adiabatic interface and conducting bottom wall and

$$
T=x-\frac{M a}{48}\left(3 y^{4}+2 y^{3}-\frac{3}{2} y^{2}-\frac{1}{2} y+\frac{3}{16}\right)
$$

for both boundaries behaving as conducting surfaces.

\subsection{Alternative formulation}

In some circumstances it is convenient to replace the original balance governing equations with alternate forms displaying some advantages with respect to the initial formulation. In general, these equivalent formulations can be obtained from the "primitive" equations via specific mathematical manipulations such as differentiation (or other artifices). Here, in particular, as stated in the introduction, the focus is on the possibility to replace the boundary condition at the free surface, namely, eq. (14), with a homogeneous boundary condition, that is, $\mathrm{dg}_{2} / \mathrm{dy}=0$ at $\mathrm{y}=1 / 2$.

It needs no demonstration that, in principle, this would be feasible by formally inserting the surface force directly in the momentum equation via an impulse function, i.e:

$$
\frac{\partial \underline{V}}{\partial t}+\underline{\nabla} \cdot[\underline{V} \underline{V}]+\underline{\nabla} p=\operatorname{Pr} \nabla^{2} \underline{V}+\underline{F}_{M a}
$$

with $\underline{F}_{M a}=-M a\left[\underline{\hat{i}}_{x} \cdot \underline{\nabla} T\right] \delta_{s}$ 
where $\hat{i}_{x}$ is the unit vector along the x direction and $\delta_{\mathrm{s}}$ is the just-mentioned "impulse" function (which takes value 1 on the interface and is zero elsewhere). Such approach, for instance, was successfully used by Tiwari and Nishino [46], who expressly modelled the surface tension effect as a body-force term acting on a very thin layer on the surface of a liquid bridge, namely

$$
F_{b o d y}=\sigma_{T} \frac{\partial T}{\partial x} \frac{1}{\delta r}
$$

where $\delta r$ is the thickness of the thin layer. These authors had to develop this method since their commercial software (STAR-LT) could not properly treat the Marangoni type boundary condition. Most recently, a similar implementation is due to Yano et al. [49]. Very thin volume elements (thickness about $5 \times 10^{-4} \mathrm{~mm}$ ) were placed next to the surface to simulate axisymmetric steady thermocapillary flows in a liquid bridge with a radius of $15 \mathrm{~mm}$ (which would correspond to a nondimensional amplitude of the thin layer $\delta \mathrm{r} \cong 10^{-5}$ ).

Though such a representation is correct from a mathematical point of view (and "appealing" given its simplicity), its usefulness, however, tends to be limited by the discontinuity intrinsically present in it.

Here we wish to show that this bottleneck can be circumvented if the ideal interface separating the two (gas and liquid) phases as a mathematical boundary of zero thickness, is replaced with a phasefield $\phi$ varying from 0 to 1 (a "distribution" function) through a region of the liquid whose thickness, though relatively small with respect to characteristic size of the domain, is not zero $(\mathrm{h} \leq \mathrm{y} \leq 1 / 2$ where $-1 / 2 \leq \mathrm{h}<1 / 2)$.

Indeed, by this artifice, it is possible to recast the surface force seen in eq. (20) into a corresponding volume force spread over a region of finite thickness, which no longer relies on the formal use of the impulse function (or the need to concentrate a volume force entirely into a layer with vanishing thickness). The required theoretical basis to this modus operandi can be found in the mathematical equality:

$$
\int_{-1 / 2}^{1 / 2} \underline{\hat{n}} \cdot \underline{\nabla} \phi d y=1
$$

where $\underline{\hat{n}}$ is obviously the unit vector locally perpendicular to the free interface ( $\hat{i}_{y}$ in our case). Accordingly, eq. (21) can be rewritten as:

$$
\underline{F}_{M a}=-M a\left[\underline{\hat{i}}_{x} \cdot \underline{\nabla} T\right]|\underline{\nabla} \phi|
$$

This condition, which can be seen as a peculiar implementation of the ideas at the basis of the socalled Continuum Surface Stress (CSS) models [50,51], in the present conditions for which the flow depends on the vertical coordinate only, reduces to 
$F_{M a}=-M a \phi^{\prime}$

$\phi$ ' being the first derivative of the function $\phi(y)$. Accordingly, eq. (12) can be reformulated as

$\frac{d^{3} g_{2}}{d y^{3}}=-\phi^{\prime \prime}$

\section{Solution}

In the following, we concentrate on a class of $\phi$ functions having the form:

$$
\phi=\left(y+\frac{1}{2}\right)^{n}
$$

with the exponent $\mathrm{n}$ being an arbitrary integer number in the interval $(1,+\infty)$, such that $\phi=0$ at $\mathrm{y}=$ $1 / 2$ and $\phi=1$ at $y=1 / 2$.

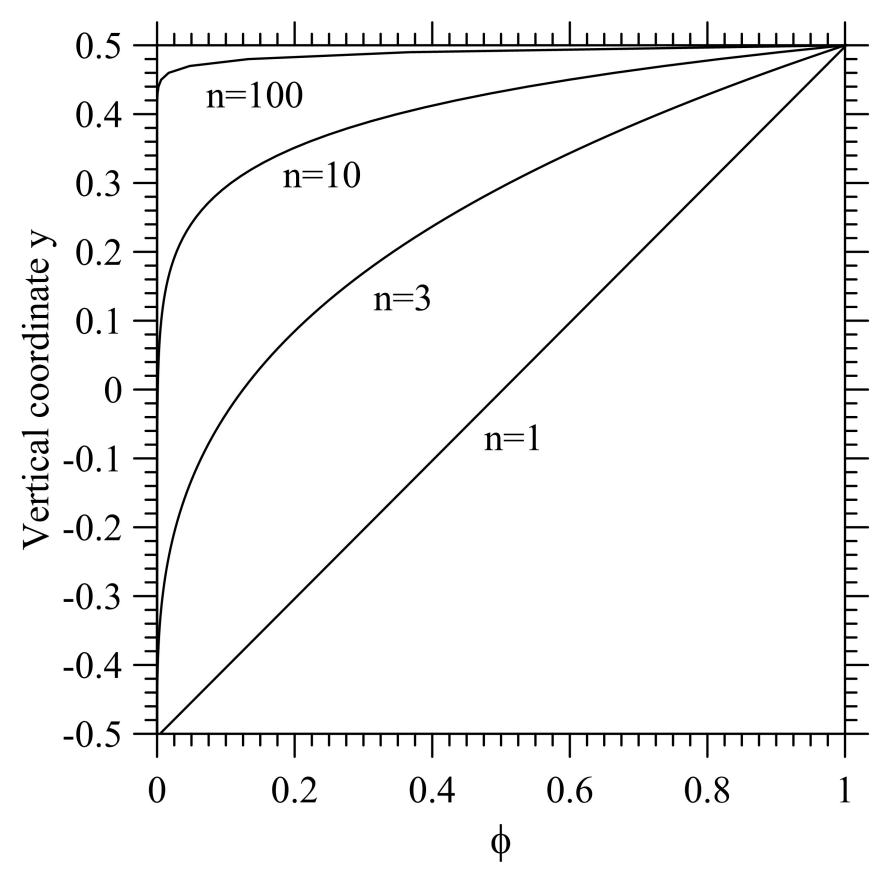

Fig. 2: Function $\phi$ as a function of $y$ for different values of the exponent $n$.

As shown in Fig. 2, this class is particularly interesting and relevant because the exponent can be used as an input parameter to set the effective amplitude of the region of finite thickness over which the volume force is spread (the limit $n \rightarrow \infty$ representing the ideal condition in which such a thickness becomes zero).

The derivative of this function yields the distribution function: 
$\phi^{\prime}=n\left(y+\frac{1}{2}\right)^{n-1}$

from which the reader will easily verify that

$$
\int_{-1 / 2}^{1 / 2} \phi^{\prime} d y=\int_{-1 / 2}^{1 / 2} n\left(y+\frac{1}{2}\right)^{n-1} d y=1
$$

and (see again Fig. 2)

$$
\lim _{n \rightarrow \infty} n\left(y+\frac{1}{2}\right)^{n-1}=\left\{\begin{array}{l}
1 \text { for } y=1 / 2 \\
0 \text { for } y<1 / 2
\end{array}\right.
$$

Moreover, by integration of eq. (25), one gets:

$$
\begin{aligned}
& \frac{d^{2} g_{2}}{d y^{2}}=-\phi^{\prime}=-n\left(y+\frac{1}{2}\right)^{n-1}+C_{1} \\
& \frac{d g_{2}}{d y}=-\left(y+\frac{1}{2}\right)^{n}+C_{1} y+C_{2} \\
& g_{2}(y, n)=-\frac{1}{n+1}\left(y+\frac{1}{2}\right)^{n+1}+\frac{1}{2} C_{1} y^{2}+C_{2} y+C_{3}
\end{aligned}
$$

where the constants $\mathrm{C}_{1}, \mathrm{C}_{2}$ and $\mathrm{C}_{3}$ can be determined considering the conditions:

solid boundary: $\mathrm{u}=0 \rightarrow \mathrm{g}_{2}(\mathrm{y})=0$

free surface: stress free condition $\rightarrow \frac{d g_{2}}{d y}=0$

no net mass flow: $\int_{-1 / 2}^{1 / 2} g_{2} d y=0$

Solution of the related system of algebraic equations yields:

$$
C_{1}=3\left[\frac{1}{2}-\frac{1}{(n+1)(n+2)}\right]
$$


$C_{2}=\left[\frac{1}{4}+\frac{3}{2} \frac{1}{(n+1)(n+2)}\right]$
$C_{3}=\left[\frac{9}{8} \frac{1}{(n+1)(n+2)}-\frac{1}{16}\right]$
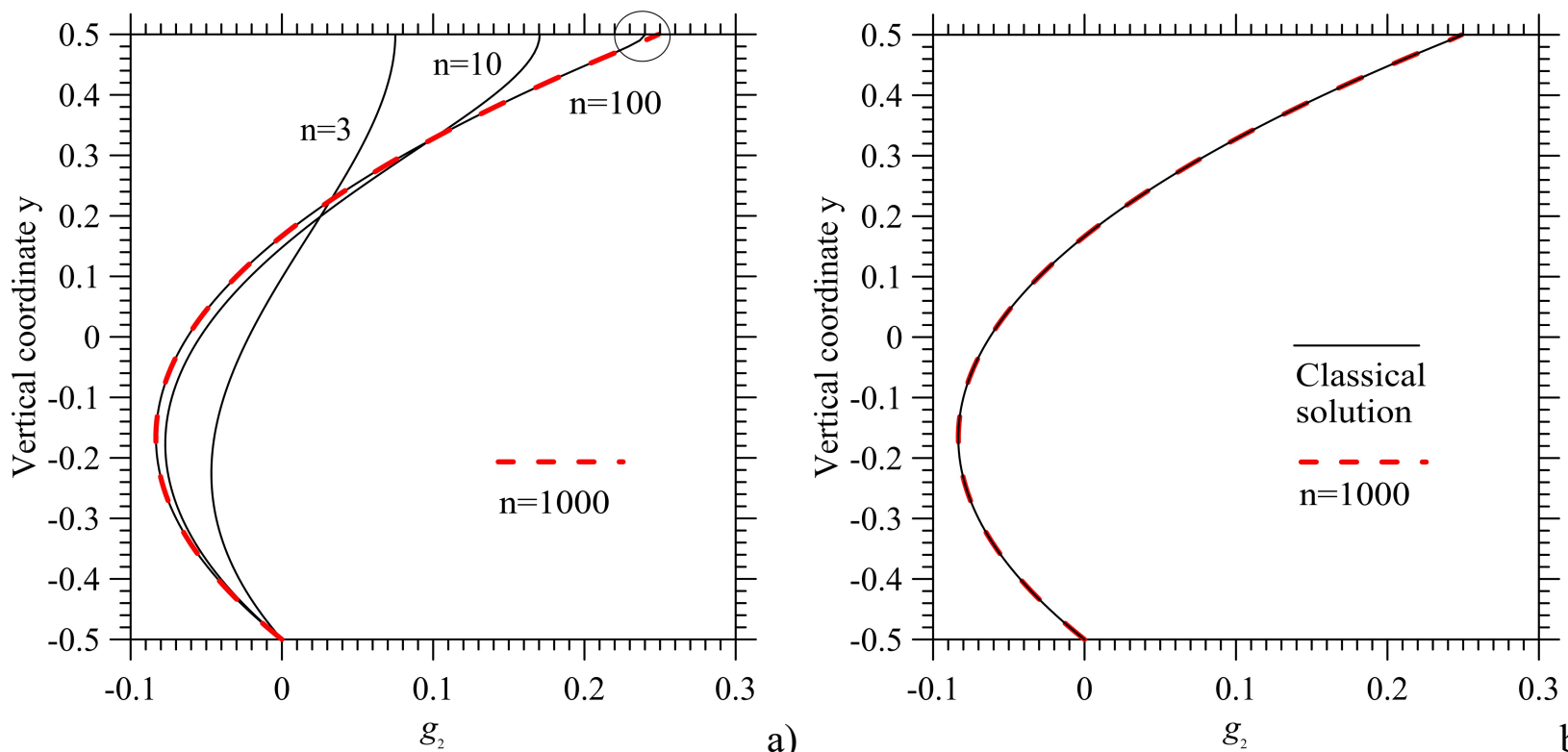

a)

$g_{2}$

b)

Fig. 3: Comparison among the velocity profiles for different values of the exponent $n$ (Fig. 3a), and the classical analytical solution by Birikh (1966) (Fig. 3b).

This class of solutions has been plotted in Fig. 3a for different values of the exponent and in Fig. 3b for $n=10^{3}$ in conjunction with the canonical analytic solution by Birikh [7] derived in Sect. 2.2.

As the reader will easily realize, the difference between the present solution and eq. (18) can be considered negligible when $n$ exceeds a given threshold $(n \geq 200$, as an example, for $n=100$ some departure can be still noticed in proximity to the top wall as evidenced by the small circle in Fig. 3a). The corresponding analytic solutions for the temperature profile can be determined by substituting $\mathrm{g}_{2}(\mathrm{y})$ into eq. (11) and considering the relevant boundary conditions for the temperature, namely, eqs. (15) or (16) according to whether one is interested in an adiabatic or conducting surface. Straightforward integration of Eq. (11) leads to $T=x+M a f_{2}(y)$ where

$f_{2}(y, n)=-\frac{1}{(n+1)(n+2)(n+3)}\left(y+\frac{1}{2}\right)^{n+3}+\frac{1}{24} C_{1} y^{4}+\frac{1}{6} C_{2} y^{3}+\frac{1}{2} C_{3} y^{2}+C_{4} y+C_{5}$

with

$$
\begin{aligned}
& C_{4}=\frac{1}{2^{4}}\left[\frac{5}{(n+1)(n+2)}-\frac{1}{2}\right] \\
& C_{5}=\frac{1}{2^{7}}\left[\frac{7}{(n+1)(n+2)}-\frac{5}{6}\right]
\end{aligned}
$$


for adiabatic interface and conducting bottom wall (Fig. 4), and

$$
\begin{aligned}
& C_{4}=\frac{1}{(n+1)(n+2)(n+3)}-\frac{1}{3} \frac{1}{2^{5}}\left[1+\frac{6}{(n+1)(n+2)}\right] \\
& C_{5}=\frac{1}{2(n+1)(n+2)(n+3)}-\frac{1}{2^{7}}\left[\frac{17}{(n+1)(n+2)}-\frac{1}{2}\right]
\end{aligned}
$$

for both boundaries behaving as conducting surfaces (Fig. 5).
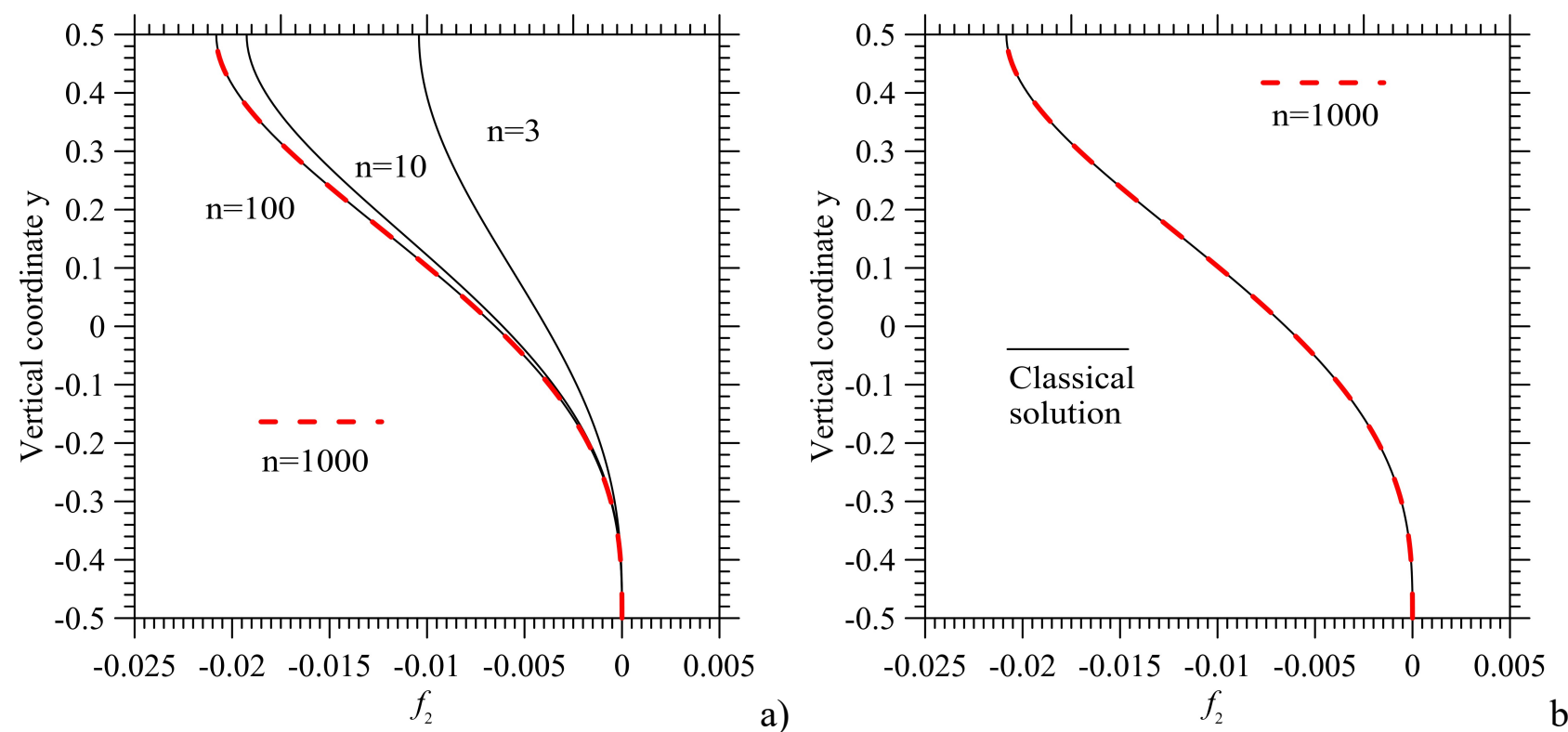

Fig. 4: Comparison among the temperature profiles for different values of the exponent $n$ (Fig. 4a), and the classical analytical solution by Birikh (1966) (Fig. 4b) in the case of adiabatic surface and conducting bottom wall).
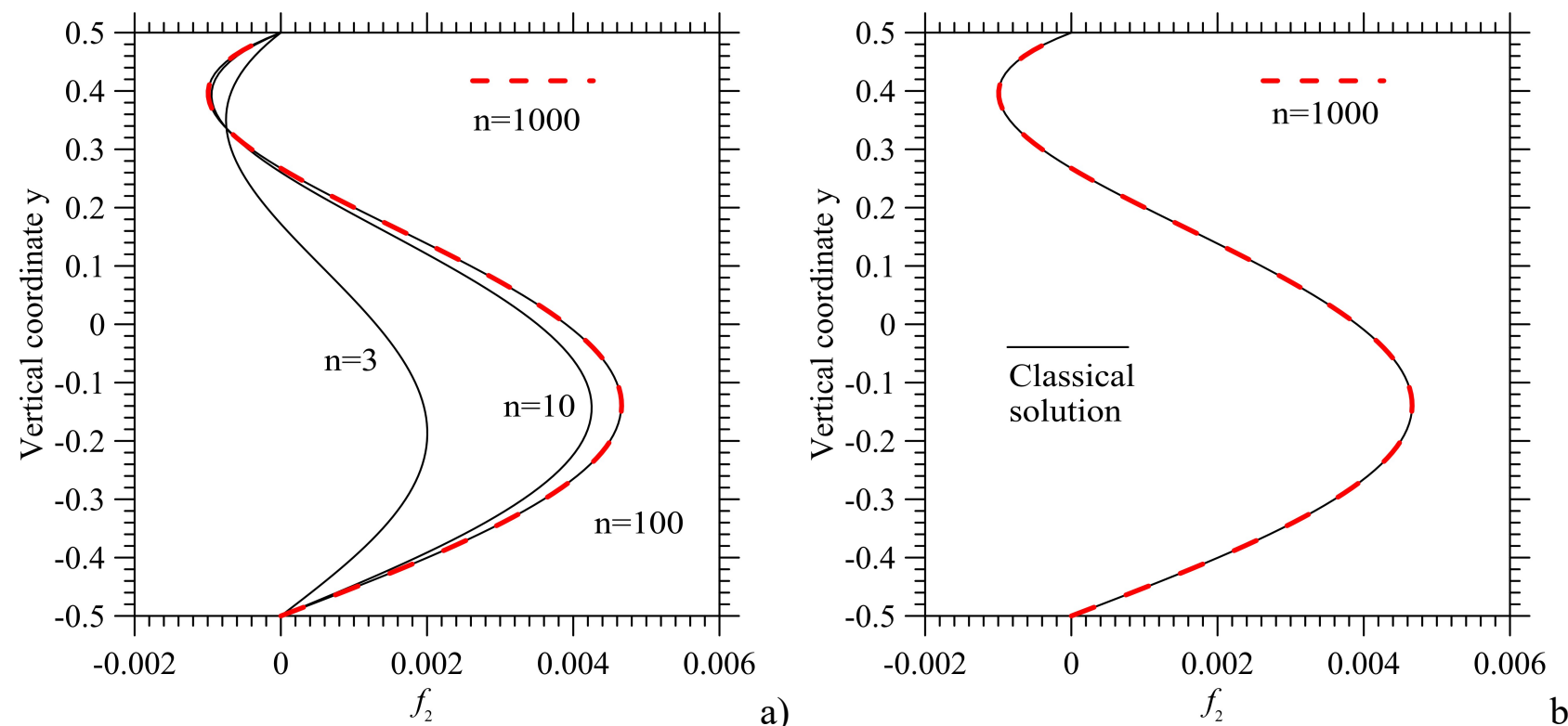

Fig. 5: Comparison among the temperature profiles for different values of the exponent $n$ (Fig. 5a), and the classical analytical solution by Birikh (1966) (Fig. 5b) in the case of both boundaries behaving as conducting surfaces. 
Additional meaningful information can be gathered from Fig. 6 in which we have plotted the following integral quantity:

$$
\theta=\int_{-1 / 2}^{1 / 2}\left|f_{2}(y, n+1)-f_{2}(y, n)\right| d y
$$
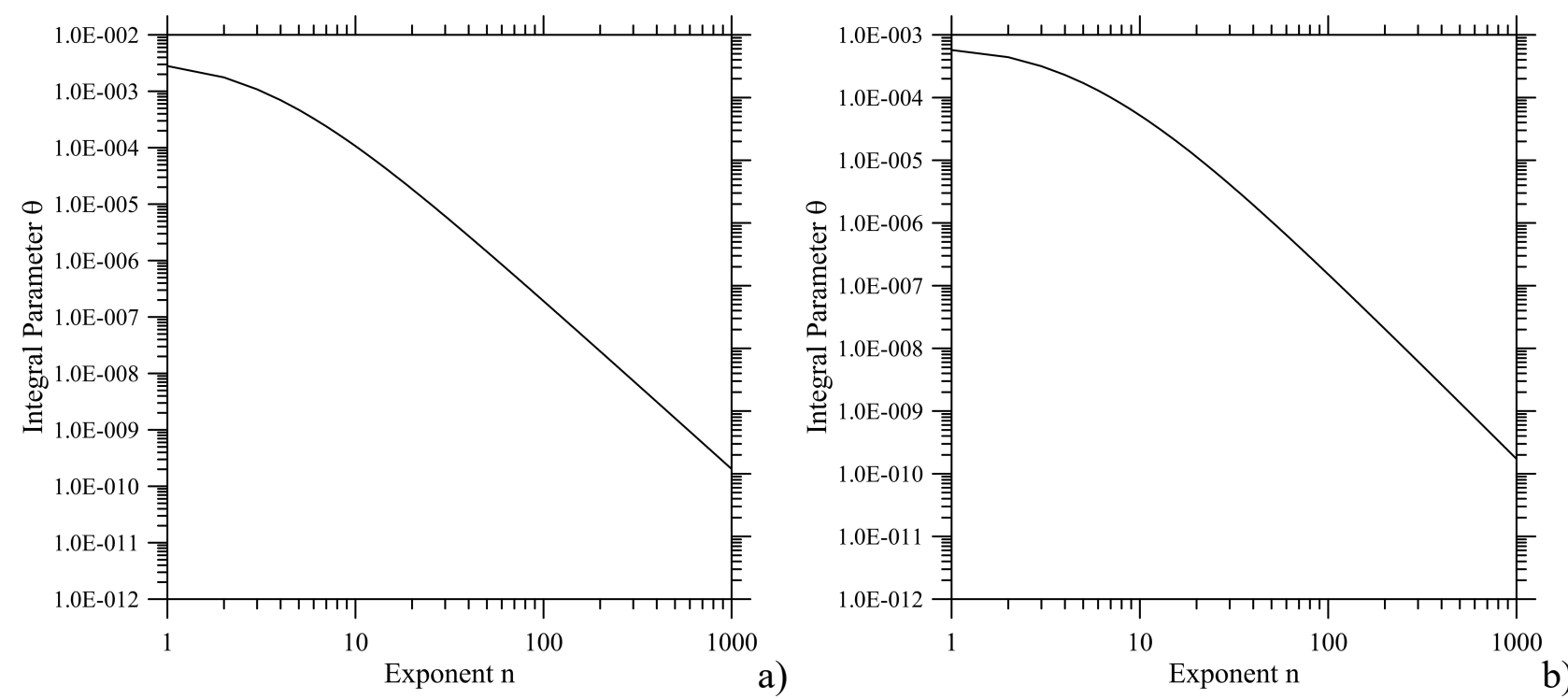

Fig. 6: Integral quantity $\theta$ defined by eq. (40) as a function of the parameter $n$ : a) adiabatic surface and conducting bottom wall; b) both boundaries behaving as conducting surfaces.

The most remarkable and intuitive consequence of such plots is that even though the proposed approach makes the analytical solution dependent on the parameter $\mathrm{n}$, the "difference" (measured by a proper integral quantity such as $\theta$ ) between the solutions corresponding to two close values of $\mathrm{n},(\mathrm{n})$ and $(\mathrm{n}+1)$, decreases as $\mathrm{n}$ increases (becoming exactly zero in the limit as $\mathrm{n} \rightarrow \infty$, which from a physical point of view would be equivalent to the size of the region with the volume force tending to zero). From an engineering (or "practical") point of view, this means that once a family of ndependent solutions has been determined through the process of analytic integration, in order to filter out the indetermination produced by the process of replacing a surface force with a volume force, it would be sufficient to consider the value of $\mathrm{n}$ for which the quantity $\theta$ becomes smaller than a given threshold or desired precision.

In order to test the general effectiveness of the overall approach based on the replacement of the surface force with an equivalent "dilute" volume force spread over a region of finite thickness (such that its integral over such a region is mathematically equal to the original surface force), we also performed some numerical simulations based on a collocated method (finite differences).

Though, as stated in the introduction, the problem related to the stability of Marangoni flow largely relies on the theory of stability and the theory of bifurcation as relevant strategies of attack, computational fluid dynamics (CFD) should also be regarded as a valid means to address it. Indeed, 
direct numerical discretization and solution of the Navier-Stokes equations in their original nonlinear and time-dependent form can provide useful information [17-33].

In particular, we used a projection method, that is, a classical numerical technique having its theoretical foundation in the Ladyzhenskaya decomposition theorem [52,53] (see, e.g., Harlow and Welch [54], Chorin [55] and Temam [56]). As usual with such a class of methods, some staggered arrangement of the variables is required (typically the fluxes and velocities are computed on the cell faces whereas other scalar variables such as temperature and pressure, occupy the center). In such implementation, we used centered finite-difference schemes with second-order accuracy for the spatial discretization of both diffusive and convective terms in the momentum equation, replacing it with a third-order accurate (quick) scheme for the convective term of the temperature equation.

These simulations have been specifically devoted to assess whether the resulting approach with the surface force spread over a region of finite thickness can provide reliable information on the typical instabilities of the Marangoni parallel flow that emerge when the characteristic number (Ma) exceeds a given threshold.

In order to mimic numerically the conditions corresponding to a parallel flow, we have considered initially two-dimensional Marangoni convection in a shallow rectangular layer of silicone oil $(\mathrm{Pr}=15)$ with aspect ratio (length/depth) $\mathrm{A}=20$ for which the flow occurring in the "core" of the cavity is sufficiently far away from the end regions (where the fluid turns around), not to be influenced significantly by such edge effects.

Though we recommend the reader to consult [31] for additional information about our algorithm and its reliability, the main results of a validation and grid refinement process are summarized here as follows. Mesh independence has been obtained with a mesh $600 \times 30$ (see Table I) to be considered as the "minimum" requirement to be satisfied in terms of grid density.

\begin{tabular}{rl}
\hline \hline Grid $\mathrm{N}_{\mathrm{x}} \times \mathrm{N}_{\mathrm{y}}$ & $\Omega_{\mathrm{HTW}}$ \\
\hline \hline $200 \times 20$ & 49.5 \\
$400 \times 20$ & 47.3 \\
$300 \times 30$ & 46.2 \\
$400 \times 30$ & 45.4 \\
$600 \times 30$ & 45.3 \\
\hline \hline
\end{tabular}

Table I: Grid Refinement Study: Angular frequency of the Hydrothermal Wave as a function of mesh resolution $\left(\operatorname{Pr}=15, \mathrm{~A}=20, \mathrm{Ma}=\sigma_{\mathrm{T}} \gamma \mathrm{d}^{2} / \mu \alpha=10^{3}\right.$, Marangoni effect implemented as boundary condition).

Validation has been achieved through comparison with the numerical results of $\mathrm{Xu}$ and Zebib [21] for pure Marangoni flow in a rectangular cavity with $\mathrm{A}=20, \operatorname{Pr}=10$ and $\mathrm{Ma}=525$. Using the same grid density that, according to the refinement study shown in Table I, guarantees a meshindependent solution (for even larger values of the Prandtl and Marangoni number), we have 
obtained a traveling-wave angular frequency $\omega \cong 36$ matching with a reasonable approximation $(\cong 2 \%)$ the value (35.17) reported by these authors.

The results of the simulations for $\operatorname{Pr}=15, \mathrm{~A}=20$ and $\mathrm{Ma}=10^{3}$ reported in Figs. 7 and 8 in terms of thermocouple signals measured at a selected point and velocity fields (in terms of streamfunction maps), do confirm that the problem is self-consistent, i.e. the oscillatory solution emerging as a result of a Hopf bifurcation (the "first" flow bifurcation in the general hierarchy of instabilities of this kind of flow) and the related frequency correctly tend, when $\mathrm{n}$ increases, to those obtained with the classical algorithm with the Marangoni force accounted for as a boundary condition (see also Fig. 9). These figures also reveal that in the opposite limit in which $n \rightarrow 1$ (namely, relatively small values of the exponent $\mathrm{n}$ ) numerical simulation are not able to capture correctly the dynamics.

It is known that the typical supercritical state of Marangoni flow for high-Pr fluids should consist of a series of convective rolls which travel continuously from the cold side to the hot side, i.e. in the upstream direction (upstream travelling wave [40]).

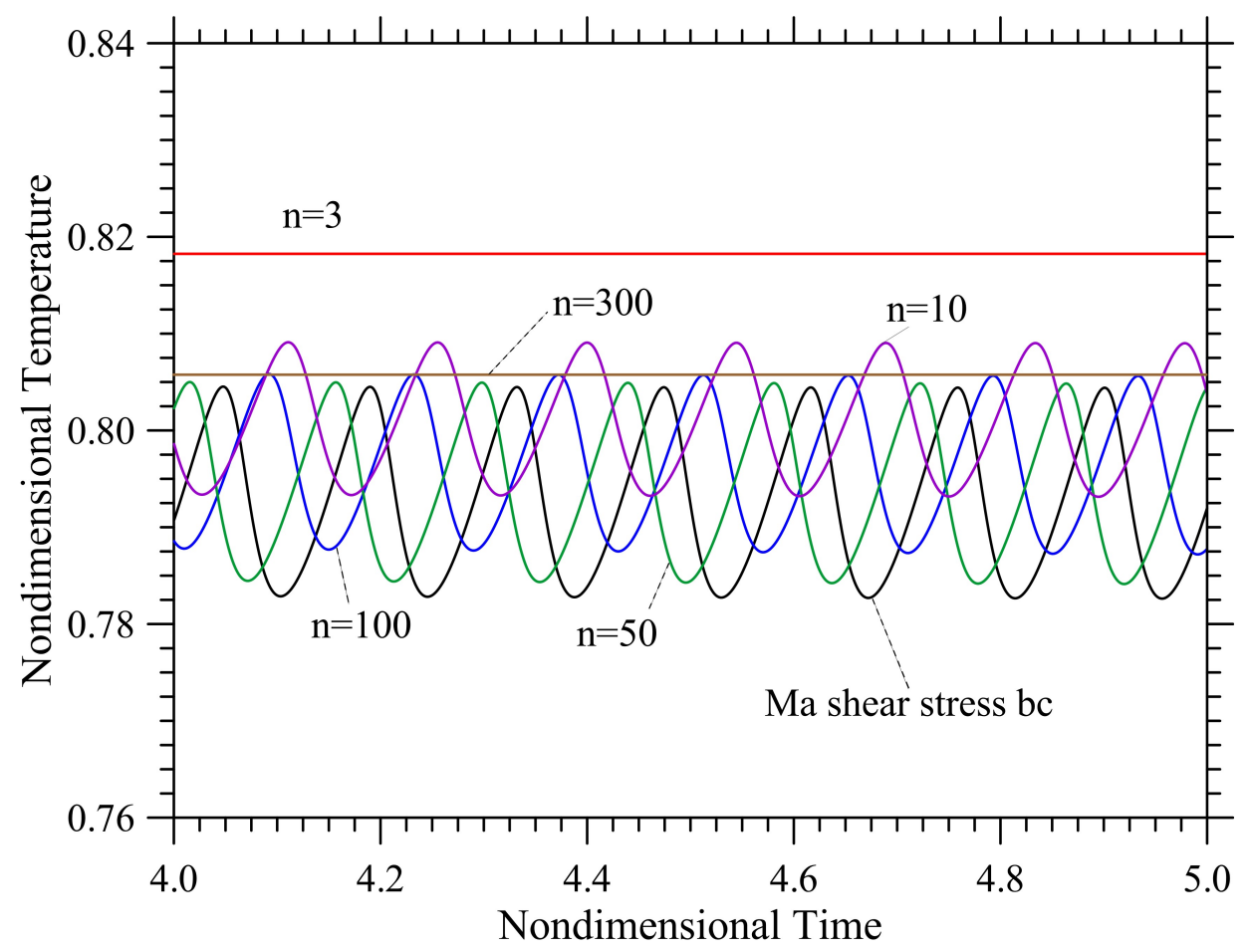

Fig. 7: Signals provided by a numerical probe located in proximity to the free surface for different values of the exponent $\mathrm{n}\left(\mathrm{N}_{\mathrm{x}} \mathrm{xN}=600 \mathrm{x} 55\right.$, layer with aspect ratio $\mathrm{A}=20, \operatorname{Pr}=15, \mathrm{Ma}=\sigma_{\mathrm{T}} \gamma \mathrm{d}^{2} / \mu \alpha=10^{3}$, adiabatic surface, conducting bottom).

As shown in Fig. 8, though for $\mathrm{n} \geq 10$ such phenomena can be correctly reproduced by the numerical simulation, for $\mathrm{n}=3$ the emerging flow is steady with a single elongated cell along the horizontal direction, similar to that that is typically established in subcritical conditions.

Moreover, though the convection amplitude obtained for $n=50$ (Fig. 8c) differs by less than $2 \%$ with respect to the classical case in which the Marangoni effect is implemented via the shear stress 
balance boundary condition (reported for completeness in Fig. 8f), for $n=10$ (Fig. 8b) some nonnegligible difference can be noticed.

The opposite limiting condition $n \rightarrow \infty$, however, also deserves some attention. Indeed, the effect of the exponent $\mathrm{n}$ in a numerical environment (or framework) where the equations are discretized and solved by a numerical technique is not as straightforward as one would imagine.
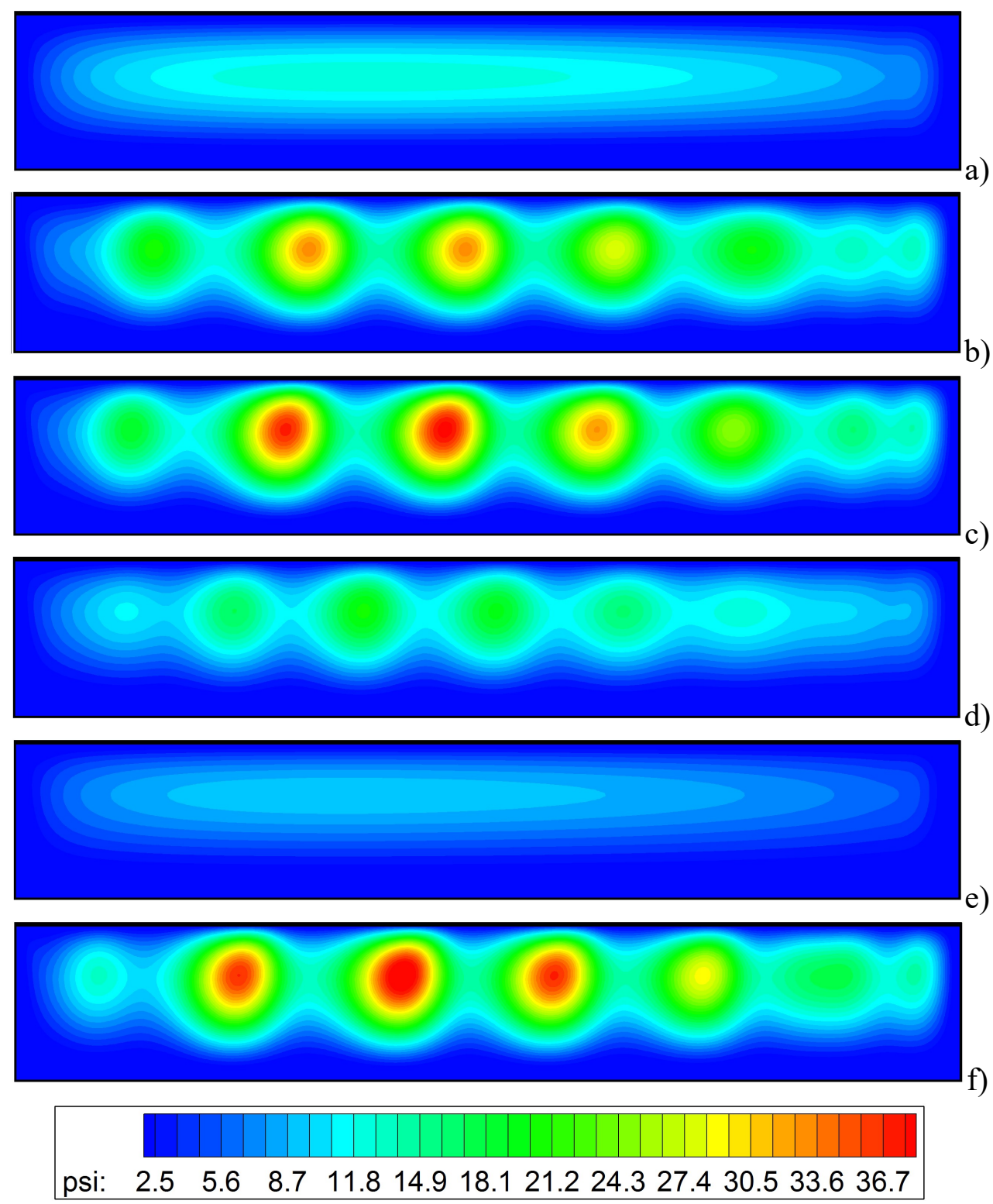

Fig. 8: Streamlines for different values of the exponent $\mathrm{n}\left(\mathrm{N}_{\mathrm{x}} \mathrm{x} \mathrm{N}_{\mathrm{y}}=600 \mathrm{x} 55\right.$, layer with aspect ratio $\mathrm{A}=20, \operatorname{Pr}=15, \mathrm{Ma}=\sigma_{\mathrm{T}} \gamma \mathrm{d}^{2} / \mu \alpha=10^{3}$, adiabatic surface, conducting bottom): a) $\left.\left.\mathrm{n}=3, \mathrm{~b}\right) \mathrm{n}=10, \mathrm{c}\right) \mathrm{n}=50$, d) $n=200$, e) $n=300$, f) Marangoni effect implemented via the classical shear stress balance boundary condition. 


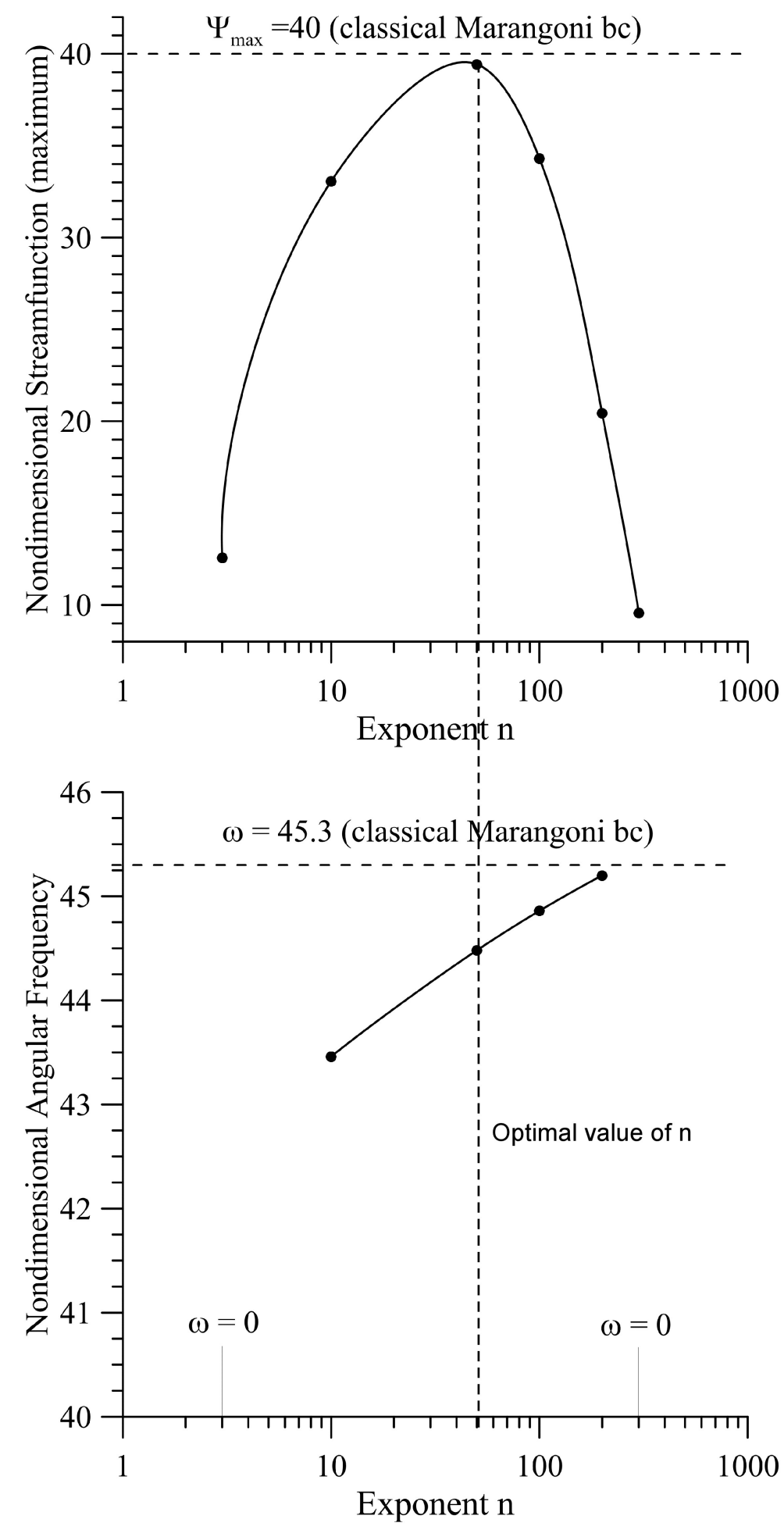

a)

b)

Fig. 9: Numerically determined maximum value of the streamfunction (a) and angular frequency (b) as a function of the exponent $\mathrm{n}$ (resolution used $\mathrm{N}_{\mathrm{x}} \mathrm{x} \mathrm{N}_{\mathrm{y}}=600 \times 55$, layer with aspect ratio $\mathrm{A}=20$, $\operatorname{Pr}=15, \mathrm{Ma}=\sigma_{\mathrm{T}} \gamma \mathrm{d}^{2} / \mu \alpha=10^{3}$, adiabatic surface, conducting bottom). 
Even though the distribution-function approach in the limit as $n \rightarrow \infty$ is still conservative in the mean, however, (as explained before) the numerical simulations have been conducted with a collocated method, which makes the algorithm not conservative in a 'discrete' sense (as a result, e.g., the flow experiences a weaker driving for $\mathrm{n}=300$ than for $\mathrm{n}=200$, see Fig. 9a). This limit becomes particularly evident in Fig. 7 where the temperature signal for $n=300$ shows no obvious evidence of the expected oscillatory flow (just as in the case $n=3$ ). Similarly, Figs. 8d and 8e reveal a notable "deficiency" in terms of flow amplitude for $n=200$ and $n=300$, respectively (which definitely proves that excessively high values of the exponent $\mathrm{n}$ can be the source of problems as well).

Additional insights into these apparently counter-intuitive behaviors can be obtained by taking into account at the same time the dependence (on the exponent $\mathrm{n}$ ) displayed by both the angular frequency $\omega$ of the emerging hydrothermal wave and its "strength" (as measured by the maximum value of the streamfunction $\psi_{\max }$ ). As illustrated in Fig. 9 for the case of a mesh $\mathrm{N}_{\mathrm{x}} \mathrm{x} \mathrm{N}_{\mathrm{y}}=600 \mathrm{x} 55$, while $\omega$ undergoes a smooth monotone increase with $n$ until it drops abruptly to zero when $n=300$, the corresponding trend of the streamfunction is not monotone. Starting from a value $\psi_{\max } \cong 12$ for $\mathrm{n}=3$ it raises until it attains a maximum for $\mathrm{n}=50$ and then its magnitude decreases again if $\mathrm{n}$ is further increased (because when such a threshold is exceeded sub-grid scales being related to the steep variations of $\phi$ can no longer be transferred to the collocated grid).

Such observations lead to the important conclusion that for a given mesh, in general, an "optimal" value of the exponent $\mathrm{n}$ can be identified providing the best agreement between the solutions obtained with the Marangoni effect modeled as a surface or volume force, respectively.

For the considered grid $\mathrm{N}_{\mathrm{x}} \mathrm{x} \mathrm{N}_{\mathrm{y}}=600 \mathrm{x} 55$ such an exponent is $\mathrm{n} \cong 50$, as witnessed by the percentage differences between the values of $\omega$ and $\psi_{\max }$ and the corresponding values obtained with the standard boundary condition, which drop below $3 \%$ for such a specific value of $n$.

Remarkably, the above considerations imply that, with the present approach, an additional requisite of numerical nature must be satisfied with respect to the situation in which the determination of analytic solutions is attempted: namely the region where the driving force is spread must be properly "resolved".

Following up on the previous point, similar criteria can be introduced by fixing the value of $n$ and allowing the number of points $\mathrm{N}_{\mathrm{y}}$ used to discretize the equation along the direction perpendicular to the liquid-gas interface to change in order to capture previously unresolved sub-grid scales. Indeed, in general, for each value of $\mathrm{n}$ a limit value of $\mathrm{N}_{\mathrm{y}}$ can be identified such that if $\mathrm{n}$ is increased while keeping constant $\mathrm{N}_{\mathrm{y}}$, the resulting solution underestimates the flow amplitude. Such a value would correspond to the optimal (minimum) number of points to be used in the vertical direction to make the numerical solution based on the spread-force approach mesh independent.

Another way to think about the above requirement is to consider that the number of grid points in the region where the variable $\phi$ undergoes significant changes (Fig. 2) must not be smaller than $a$ given "minimum". As such a region undergoes significant shrinkage as $\mathrm{n}$ is increased, the number of points in the vertical direction has to be increased accordingly (this being equivalent to a minimum of $\mathrm{N} \cong 5$ points uniformly distributed in such a region for the values of the Prandtl and 
Marangoni number considered here). Obviously, such a requirement would lead to the need of an infinite number of grid points in the vertical direction in the limit as $n \rightarrow \infty$. Practically speaking, this means a critical aspect being related to the application of the present framework is the just discussed identification of a compromise between the two opposite needs to put the code in a condition to capture qualitatively and quantitatively (with an acceptable approximation) the dynamics of interest and not increase excessively the related computational cost.

As the reader might have realized at this stage, keeping in mind the above considerations and general criteria, these sample results might be seen as the required basis to exploit the suggested approach to model surface-tension effects in existing commercial or open-source CFD codes for which a major shortcoming is represented by the inability of the software to accept nonhomogeneous Neumann conditions for the velocity.

As an additional example, the ability of the present framework to reproduce typical dynamics of surface-tension driven flows even in circumstances for which the temperature gradient is perpendicular to the free surface (the classical three-dimensional Marangoni-Bénard problem) is illustrated in Figs. 10 and 11.

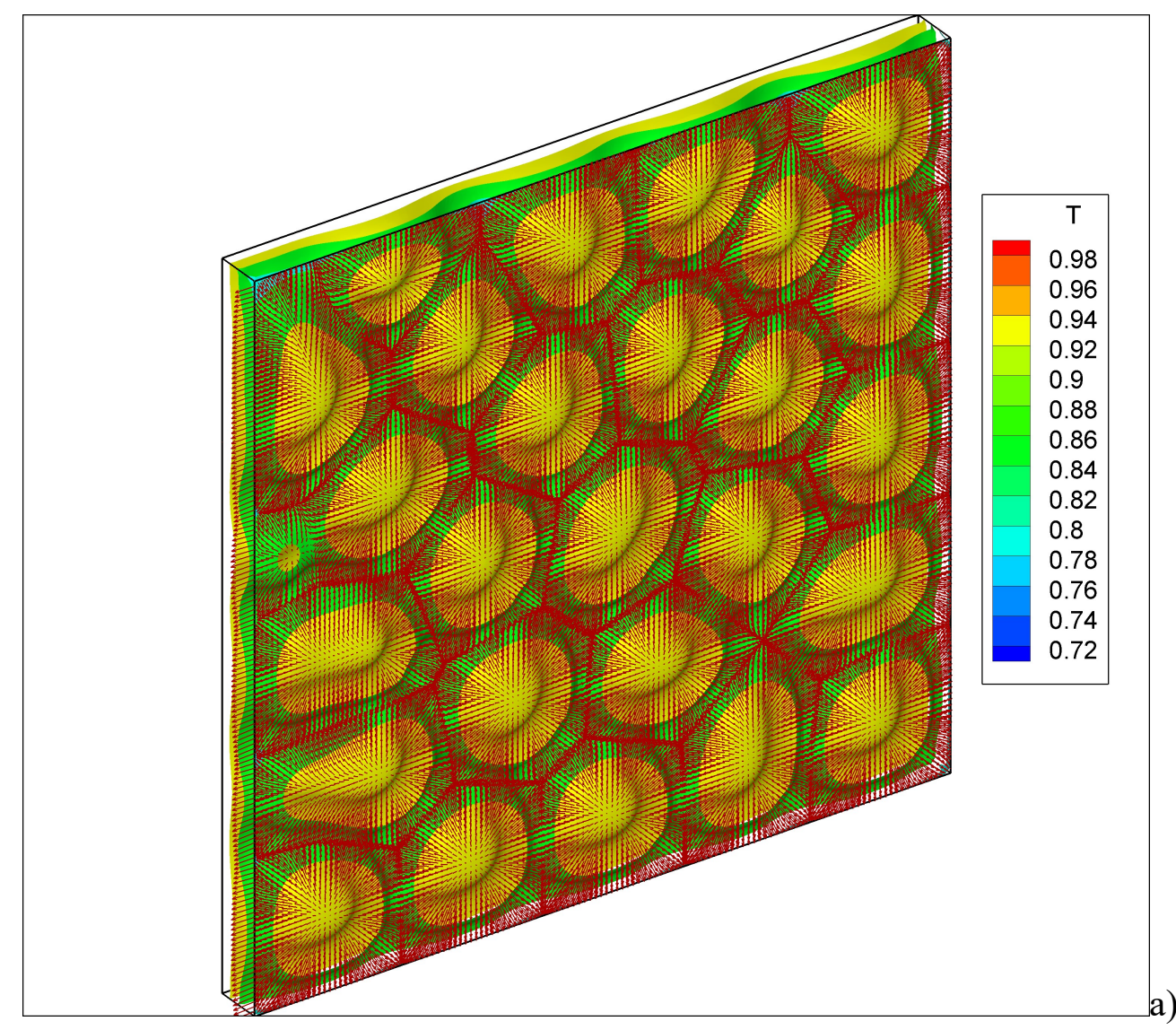




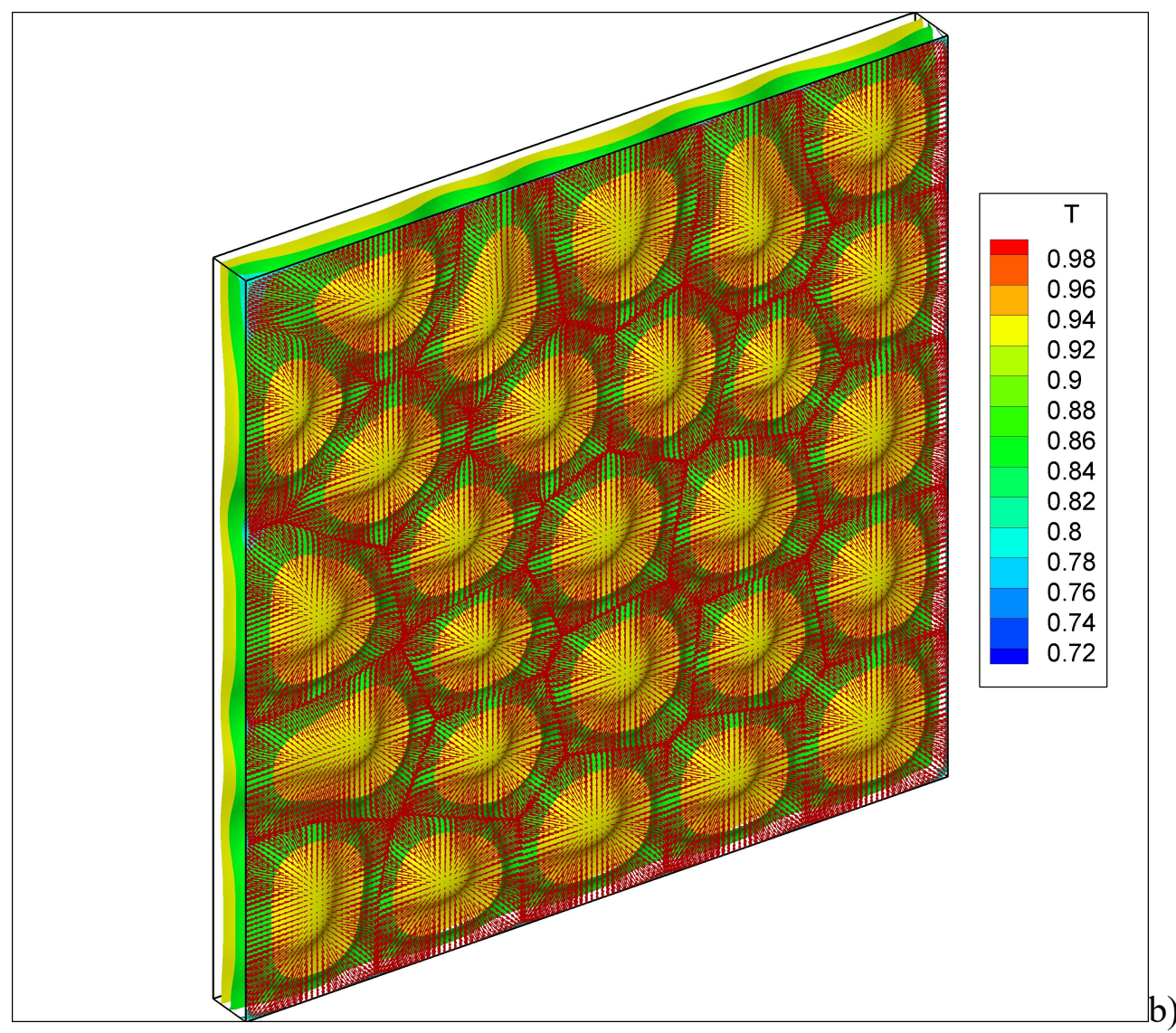

Fig. 10: Three-dimensional isosurfaces of temperature and velocity field (vector plot) at the free liquid-gas interface for Marangoni-Bénard convection in a layer of silicone oil of $1 \mathrm{cSt}(\mathrm{Pr}=15)$ covered by an equal layer of air, having nondimensional depth $\mathrm{L}_{\mathrm{z}}=1$ and horizontal dimensions $\mathrm{L}_{\mathrm{x}}=15$ and $\mathrm{L}_{\mathrm{y}}=15\left(\mathrm{Ma} \cong 180\right.$, Ma defined as $\sigma_{\mathrm{T}} \Delta \mathrm{Td} / \mu \alpha$ where $\mathrm{d}$ is the layer depth and $\Delta \mathrm{T}$ the temperature difference along the vertical direction; zero-g conditions, $\mathrm{N}_{\mathrm{x}} \mathrm{xN}_{\mathrm{y}} \mathrm{xN} \mathrm{N}_{\mathrm{z}}=300 \mathrm{x} 300 \mathrm{x} 40$ ): a) Marangoni effect implemented via the classical shear stress balance boundary condition; b) Marangoni effect implemented as volume force with $n=40$.

Figure 10 shows the classical steady hexagonal cells at the free liquid-gas interface that are typical of this kind of convection in relatively shallow layers of fluids for not too high values of the Marangoni number.

The isosurfaces of temperature in Fig. 11 provide some additional insights into the coupling between the velocity and temperature fields showing that (as expected) a rising current of hot fluid is established beneath the core of each hexagonal cell. Unlike the traveling wave solution that develops on an initially steady underlying unicellular shear flow, this type of convection originates from purely quiescent and thermally diffusive conditions when the applied temperature difference exceeds a critical value. 

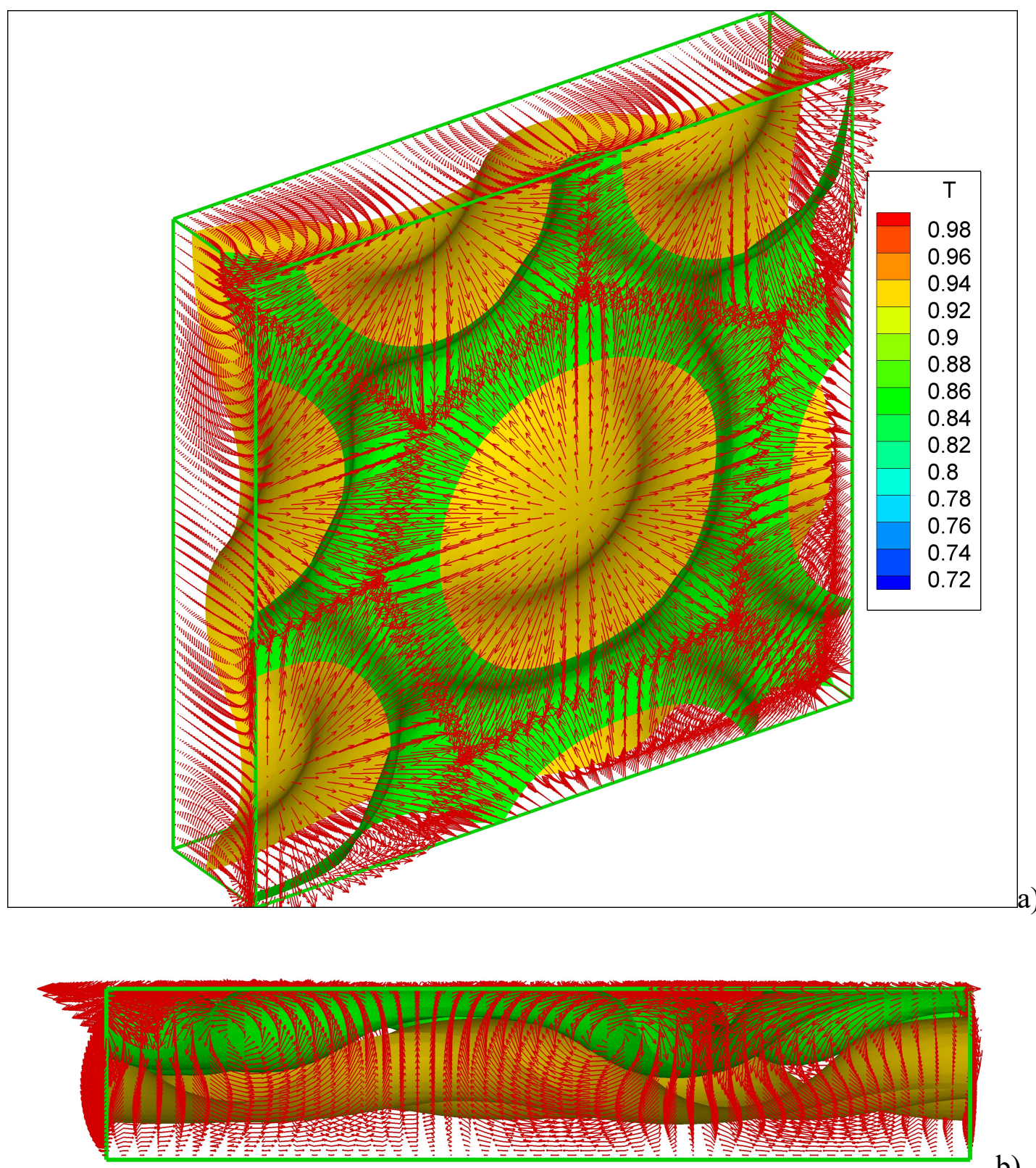

b)

Fig. 11: a) Enlarged view of the central region of the layer $(5 \leq x \leq 10,5 \leq y \leq 10)$ and b) related lateral view, for the same conditions corresponding to Fig. 10b (Marangoni effect implemented as volume force with $\mathrm{n}=40$ ).

Comparison between Fig. 10a and 10b, obtained using the classical shear stress balance condition at the interface and the equivalent formulation in terms of volume force, respectively, indicates that the latter approach can capture both quantitatively and qualitatively the typical features of Marangoni-Bénard convection. Indeed, apart from some minor qualitative differences between the two patterns (essentially due to the intrinsically "random nature" of the infinitesimal disturbances leading to the onset of convection starting from purely quiescent conditions), the two solutions display the same number of cells (flow wavelength) and maximum value of velocity (intensity or strength of the flow when the initial perturbations saturate their amplitude). 


\section{Discussion and Conclusions}

The advantages resulting from the approach proposed here would be relevant to all those circumstances in which taking into account the Marangoni effect via a boundary condition might play the role of a bottleneck for further advancements.

Stripped to its basics, the proposed scheme envisions the replacement of such a boundary condition with a source term in the momentum equation properly "weighted" by means of a "phase" function: 1) having the shape of a polynomial term of order $n, 2$ ) varying between 0 and 1 over the considered domain ( 1 corresponding to the location of the interface) and 3) ideally tending to an impulse function in the limit as the exponent becomes infinite.

Even if the formalism developed in the present paper has been mainly based on the assumption of parallel flows, the resulting ideas, however, are not restricted to such a specific case as demonstrated by the examples related to the case of three-dimensional Marangoni-Bénard convection. It is likely that the intrinsic flexibility of the resulting framework will allow this research to branch out and model or simulate a whole array of different dynamics. As mentioned in the introduction, this could indeed be the case of future attempts directed to the determination of analytic solution for the case of non-Newtonian fluids. Moreover, by removing the bottleneck that the shear stress balance at the interface represents, the present research might also support the utilization of existing numerical tools where the possibility to impose non-homogeneous Neumann boundary conditions is not an option or would require substantial (non-straightforward) modifications of the tool (a typical example being represented by the Open-Foam platform).

The differences between the proposed alternate approach and the earlier implementation attempted by [46] have been illustrated and its advantages and drawbacks discussed critically.

Apart from these rather technical facts, our analysis might also have remarkable unexpected connections on a much more conceptual level with the so-called existence and uniqueness problem for the Navier-Stokes equations (Galdi [57]).

In its general form this very popular problem can be stated as follows: "Given the body force $\underline{F}_{b}$ and the initial distribution of velocity $\underline{V}_{o}=\underline{V}(\underline{r}, 0)$ (no matter how smooth), to determine a corresponding unique regular solution of velocity and pressure for all times $t>0$ ”.

This dilemma has not yet been satisfactorily settled (this one of the reasons for which sometimes it is also referred to as the "millennium problem"). Some valuable efforts towards the solution of it, however, can be found in the literature since 1933 (Leray [58-60] and [52]).

Most of these theoretical studies have been directed to the case in which the driving force is a volume force (as implicit in the problem statement per se). For the case of buoyancy flow, as an example, the interested reader may consider [61-79].

Surprisingly, however, no results specific for the case of Marangoni flow have been obtained or presented so far.

As the interplay between available numerical and analytic solutions and theoretical arguments has been particularly fruitful in the development of the above theory, pursuing a much more speculative 
impact of our ideas, it might be argued that the approach elaborated in the present short work might stimulate investigators operating in the above field to consider the possibility to reformulate the general existence and uniqueness problem for Marangoni flow solutions (regardless of the solution representation approach used, be it analytical or numerical) in the same theoretical framework already developed in the past for the case of buoyancy flow (in which the driving force is treated as a canonical "volume force").

Though the price for the process of replacing a surface force with a volume force (namely, a boundary condition with a source term in the momentum equation) is the emergence of an evident indetermination (as witnessed by the multitude of analytic solutions satisfying the governing equations when $\mathrm{n}$ is varied in the range $0<\mathrm{n}<\infty$ or the need to attain a satisfactory balance between the polynomial degree of the distribution function and the mesh space resolution when the problem is approached numerically), it could be argued that the uniqueness of the considered solution would/could be recovered in the limit as $n \rightarrow \infty$ (or in an equivalent way, the thickness of the region of existence of $\mathrm{F}_{\mathrm{Ma}} \rightarrow 0$ ). This could be a relevant way to address the general existence and uniqueness problem for the case of Marangoni convection resorting to already existing approaches and attempts for the case of buoyancy flow, such as those described in the works cited above [5779].

Future work of the present author will be concerned with the application of the present models to the case of non-Newtonian (viscoelastic) fluids for the determination of both new analytic and numerical solutions. By turning the Marangoni stress into a force volume, both viscoelastic and Marangoni forces reduce to source terms to be added to the momentum equation (with the ensuing possibility to set homogeneous boundary conditions at the free interface for both the momentum and viscoelastic stress transport equations).

\section{References}

[1] Melnikov D.E., Shevtsova V., Yano T., Nishino K., (2015), Modeling of the experiments on the Marangoni convection in liquid bridges in weightlessness for a wide range of aspect ratios, International Journal of Heat and Mass Transfer, 87 :119-127.

[2] Melnikov D.E., Shevtsova V.M., (2014), The effect of ambient temperature on the stability of thermocapillary flow in liquid column, International Journal of Heat and Mass Transfer, 74: 185195.

[3] Li K., Matsumoto S., Imaishi N., Hu W.-R., (2015), Marangoni flow in floating half zone of molten tin, International Journal of Heat and Mass Transfer, 83: 575-585.

[4] Xun B., Li K., Hu W.-R., Imaishi N., (2011), Effect of interfacial heat exchange on thermocapillary flow in a cylindrical liquid bridge in microgravity, International Journal of Heat and Mass Transfer, 54(9-10): 1698-1705.

[5] Henry D., Kaddeche S. and Ben Hadid H., (2005), Stabilization of thermogravitational flows by magnetic field and surface tension, Phys. Fluids, 17, 054106-1-054106-9.

[6] Ostroumov G.A. (1952), Free convention under the conditions of the internal problem, NACA TM, Washington, April 1958, Translation of Svobodnaya convectzia v ousloviakh vnoutrennei zadachi, State Publishing House, Technico-Theoretical Literature, Moscow-Leningrad, 1952. 
[7] Birikh R.V., (1966), "Thermocapillary convection in a horizontal fluid layer", J. Appl. Mech. Tech. Phys. 7, pp. 43-49.

[8] Birikh R.V., (1990), "Vibrational convection in a plane layer with a longitudinal temperature gradient", Fluid Dynamics, 25(4): 500-503. Translated from Izvestiya Akademii Nauk SSSR, Mekhanika Zhidkosti i Gaza, No. 4, pp. 12-15, July-August, 1990.

[9] Gershuni G.Z. and Zhukhovitskii E.M., (1979), "Free thermal convection in a vibrational field under conditions of weightlessness”, Sov. Phys. Dokl., 24(11), pp. 894-896.

[10] Belghazi H., El Ganaoui M., Labbe J.C., (2010), "Analytical solution of unsteady heat conduction in a two-layered material in imperfect contact subjected to a moving heat source", International Journal of Thermal Sciences, 49(2), pp. 311-318.

[11] Lappa M., (2012), "Exact Solutions for Thermal Problems: Buoyancy, Marangoni, Vibrational and Magnetic-Field-Controlled Flows", Review of Applied Physics, 1(1), pp. 1-14

[12] Lappa M. and Ferialdi H., (2017), "On the General Properties of Steady Gravitational Thermal Flows of Liquid Metals in Variable Cross-section Containers", Review of Applied Physics, 5(1): 110. DOI: 10.14355/rap.2017.05.001

[13] Jane B., (2007), "Exact solutions to one dimensional non-homogeneous parabolic problems by the homogeneous Adomian decomposition method", Appl. Math. Comput., 186, pp. 969-979.

[14] Jebari R., Ghanmi I. and Boukricha A., (2013), "Adomian decomposition method for solving nonlinear heat equation with exponential nonlinearity", Int. Journal of Math. Analysis, 7(15), pp. 725- 73

[15] Al-Saif A. S. J., (2015), “Analytical approximate solutions for two-dimensional incompressible Navier-Stokes equations", Advances in Physics Theories and Applications, 49, pp. 69-86.

[16] Makinde O. D. and Olanrewaju P. O. (2010) "Buoyancy Effects on Thermal Boundary Layer Over a Vertical Plate With a Convective Surface Boundary Condition”, ASME J. Fluids Eng., 132(4), 044502 (4 pages)

[17] Kuo H.P., Korpela S.A., (1988), "Stability and finite amplitude natural convection in a shallow cavity with insulated top and bottom and heated from the side", Phys. Fluids, 31, pp. 33-42.

[18] Mundrane M. and Zebib A., (1993), "Two and three dimensional buoyant thermocapillary convection", Phys. Fluids A, 5, pp. 810-818.

[19] Peltier L. and Biringen S., (1993), "Time-dependent thermocapillary convection in a rectangular cavity: numerical results for a moderate Prandtl number fluid", J. Fluid Mech., 257, pp. 339-357.

[20] Sasmal G. P., Hochstein J. I., (1994), "Marangoni Convection With a Curved and Deforming Free Surface in a Cavity”, ASME J. Fluids Eng., 116(3), pp. 577-582.

[21] Xu J. and Zebib A., (1998), Oscillatory two- and three-dimensional thermocapillary convection, J. Fluid Mech., 364, pp. 187-209.

[22] Gelfgat A.Yu., Bar-Yoseph P.Z. and Yarin A.L., (1999), "Stability of Multiple Steady States of Convection in Laterally Heated Cavities", J. Fluid Mech., 388, pp. 315-334.

[23] Delgado-Buscalioni R. and Crespo del Arco E., (2001), "Flow and heat transfer regimes in inclined differentially heated cavities”, Int. J. Heat Mass Transfer, 44, pp. 1947-1962.

[24] Tumin A., (2003), "The Spatial Stability of Natural Convection Flow on Inclined Plates", ASME J. Fluids Eng., 125(3), pp. 428-437.

[25] Shevtsova V.M., Nepomnyashchy A.A. and Legros J.C., (2003), “Thermocapillary-buoyancy convection in a shallow cavity heated from the side", Phys. Rev. E 67, 066308 (14 pages).

[26] Saghir M. Z., Makriyannis T. J. and Labrie D., (2004), "Buoyancy Convection During the Growth of SixGe1-x by the Traveling Solvent Method (TSM)", ASME J. Fluids Eng., 126(2), pp. 223-228.

[27] Bucchignani E., (2004), "Numerical characterization of hydrothermal waves in a laterally heated shallow layer", Phys. Fluids, 16(11), pp. 3839-3849.

[28] Xu B., Ai X. and Li B. Q., (2006), "Thermocapillary Instabilities of Low Prandtl Number Fluid in a Laterally Heated Vertical Cylinder”, ASME J. Fluids Eng., 128(6), pp.1228-1235. 
[29] Lappa M., (2007), Secondary and oscillatory gravitational instabilities in canonical threedimensional models of crystal growth from the melt, Part2: Lateral heating and the Hadley circulation, Comptes Rendus Mécanique, 335(5-6): 261-268.

[30] Lappa M., (2016), Control of convection patterning and intensity in shallow cavities by harmonic vibrations, Microgravity Science \& Technology, 28(1), pp. 29-39.

[31] Lappa M., (2016), "Patterning behaviour of gravitationally modulated supercritical Marangoni flow in liquid layers", Phys. Rev. E Statistical Nonlinear, and Soft Matter Physics, 93(5), 053107, (13 pages).

[32] Lappa M., (2017), Hydrothermal waves in two-dimensional liquid layers with sudden changes in the available cross-section, Int. J. Num. Meth. Heat Fluid Flow, DOI: 10.1108/HFF-11-20160453

[33] Lappa M., (2017), On the oscillatory hydrodynamic modes in liquid metal layers with an obstruction located on the bottom, Int. J. Thermal Science, 118: 303-319.

DOI: 10.1016/j.ijthermalsci.2017.04.013

[34] Lin C.-C., (1944), "On the stability of two-dimensional parallel flows", Proc. NAS, 30(10), pp. 316-324.

[35] Rosenbluth M.N. and Simon A., (1964), "Necessary and sufficient conditions for the stability of plane parallel inviscid flow", Phys. Fluids, 7(4), pp. 557-558.

[36] Drazin P. and Howard L.N., (1966), "Hydrodynamic stability of parallel flow of inviscid fluid", Adv. Appl. Mech., 9, pp. 1-89.

[37] Hart J.E., (1972), "Stability of thin non-rotating Hadley circulations", J. Atmos. Sci., 29, pp. 687-697.

[38] Hart J.E., (1983), "A note on the stability of low-Prandtl-number Hadley circulations", J. Fluid Mech., 132, pp. 271-281.

[39] Gill A. E., (1974), "A theory of thermal oscillations in liquid metals", J. Fluid Mech. 64 (3), pp. 577-588.

[40] Smith M.K., Davis S.H. (1983), "Instabilities of dynamic thermocapillary liquid layers. Part 1 : convective instabilities ", J. Fluid Mech, 132, pp. 119-144

[41] Laure P. and Roux B., (1989), "Linear and non linear study of the Hadley circulation in the case of infinite cavity", J. Cryst. Growth 97(1), pp. 226-234.

[42] Gershuni G.Z., Laure P., Myznikov V.M., Roux B., Zhukhovitsky E.M., (1992), "On the stability of plane-parallel advective flows in long horizontal layers", Microgravity Q., 2(3), pp. 141151.

[43] Parmentier P.M., Regnier V.C. and Lebon G., (1993), "Buoyant-thermocapillary instabilities in medium-Prandtl number fluid layers subject to a horizontal temperature gradient", Int. J. Heat Mass Transfer, 36, pp. 2417-2427.

[44] Priede J., Thess A., Gerbeth G., (1995), "Thermocapillary instabilities in liquid metal: Hartmann number versus Prandtl number", Magnetohydrodynamics, 31(4), pp. 420-428.

[45] Priede J. and Gerbeth G., (1997a), "Influence of thermal boundary conditions on the stability of thermocapillary-driven convection at low Prandtl numbers", Phys. Fluids, 9, pp. 1621-1634.

[46] Tiwari S. and Nishino K., (2007), Numerical study to investigate the effect of partition block and ambient air temperature on interfacial heat transfer in liquid bridges of high Prandtl number fluid, J. Cryst. Growth, 300:486-496.

[47] Lappa M., Thermal Convection: Patterns, Evolution and Stability (John Wiley \& Sons, Chichester, England, 2009).

[48] Lappa M., Rotating Thermal Flows in Natural and Industrial Processes (John Wiley \& Sons, Chichester, England, 2012).

[49] Yano T., Maruyama K., Matsunaga T. and Nishino K., (2016), Effect of ambient gas flow on the instability of Marangoni convection in liquid bridges of various volume ratios, Int. J. Heat Mass Transf., 99: 182-191. 
[50] Gueyffier, D.; Li, J., Nadim, A.; Scardovelli, S.; Zaleski, S., (1999): Volume of Fluid interface tracking with smoothed surface stress methods for three-dimensional flows. J. Comput. Phys.,. 152, pp. 423-456

[51] Lappa M., (2005), "Coalescence and non-coalescence phenomena in multi-material problems and dispersed multiphase flows: Part 2, a critical review of CFD approaches", Fluid Dynamics \& Materials Processing, 1(3), pp. 213-234.

[52] Ladyzhenskaya O.A., (1969), "The Mathematical Theory of Viscous Incompressible Flow", Gordon and Breach, $2^{\text {nd }}$ Edition, New York - London, 1969.

[53] Lappa M., (2016), On the onset of multi-wave patterns in laterally heated floating zones for slightly supercritical conditions, Physics of Fluids, 28(12): 124105 (22 pages).

[54] Harlow F.H. and Welch J.E. (1965), "Numerical calculation of time-dependent viscous incompressible flow with free surface", Phys. Fluids, 8, pp. 2182-2189.

[55] Chorin A.J., (1968), "Numerical solutions of the Navier-Stokes equations", Math. Comput., 22, pp. 745-762.

[56] Temam R., (1968), "Une méthode d'approximation de la solution des équations de NavierStokes", Bull. Soc. Math. France, 98, pp. 115-152.

[57] Galdi G.P., (2000), "An introduction to the Navier-Stokes initial-boundary value problem", Fundamental directions in mathematical fluid mechanics/Giovanni P. Galdi ed.,-Basel; Boston; Berlin: Birkhauser 2000, pp.1-70.

[58] Leray J., (1933), "Etude de diverses équations intégrales nonlinéaires et de quelques problemes que pose l'hydrodinamique", J. Math. Pures Appl., 12, pp. 1-82.

[59] Leray J., (1934a), "Sur le mouvement d'un fluide visqueux emplissant l'espace", Acta Math., 63, pp. 193-248.

[60] Leray J., (1934b), "Essai sur les Mouvements Plans d'un Liquide Visqueux que Limitent des Parois", J. Math. Pures Appl., 13, pp. 331-418.

[61] Rabinowitz P.H., (1968) "Existence and nonuniqueness of rectangular solutions of the Bénard problem", Arch. Rat. Mech. Anal., 29, pp. 32-57.

[62] Fife P.C. and Joseph D.D., (1969), "Existence of convective solutions of the generalized Bénard problem which are analytic in their norm", Arch. Rat. Mech. Anal., 33(2), pp. 116-138.

[63] Cannon J.R. and DiBenedetto E., (1980), "The initial value problem for the Boussinesq equations with data in Lp", Approximation methods for Navier-Stokes problems, 129-144, Lecture Notes in Math., 771, Springer, Berlin, 1980.

[64] Morimoto H., (1989), "On the existence of weak solutions of equation of natural convection", J. Fac. Sci. Univ. Tokyo, Sect. IA, 36, pp. 87-102.

[65] Morimoto H., (1991), "On the existence and the uniqueness of weak solutions of equations of natural convection”, Tokyo J. Math., 14, pp. 217-226.

[66] Fang Y.-f. and Grillakis M.G., (1996), "Existence and Uniqueness for Boussinesq type equations on a circle", Comm. Partial Diff. Eq., 21(7 \& 8), pp. 1253-1277.

[67] Skalák Z. and Kucera P., (2000), "An existence theorem for the Boussinesq equations with non-Dirichlet boundary conditions", J. Appl. Math., 45(2), pp. 81-98.

[68] da Rocha M.S., Rojas-Medar M.A.; Rojas-Medar M.D., (2003), "On the existence and uniqueness of the stationary solution to equations of natural convection with boundary data in $\mathrm{L}^{2 \text { ", }}$ Proc. Roy. Soc. Lond., A 459(2031), pp. 609-621.

[69] Danchin R., (2003), "Density-dependent incompressible viscous fluids in critical spaces", Proceedings of the Royal Society of Edinburgh, 133A, pp. 1311-1334.

[70] Ladyzhenskaya O.A., (2003), "Sixth problem of the millennium: Navier-Stokes equations, existence and smoothness", Russ. Math. Surv., 58(2), pp. 251-286.

[71] Shilkin T.N., (2004), "Partial Regularity of Weak Solutions of the Stationary 3D-Boussinesq System”, J. Math. Sci., 123(6), pp. 4668-4677

[72] González F.G., da Rocha M.S., and Medar M.R., (2005), "The Cauchy problem and decay rates for strong solutions of a Boussinesq system", Abstract and Applied Analysis, 7, pp. 757-766. 
[73] Villamizar-Roa E.J., Rodríguez-Bellido M.A., and Rojas-Medar M.A., (2006), "The Boussinesq system with mixed nonsmooth boundary data", Comptes Rendus Mathematique, 343(3), pp. 191-196.

[74] Naumann J., (2006), "On the existence of weak solutions to the equations of non-stationary motion of heat-conducting incompressible viscous fluids", Math. Meth. Appl. Sci., 29(16), pp.18831906.

[75] Kaizu S. and Saito J., (2007), "Boussinesq Equations in Three Dimensional Thin Domains and the Corresponding Two Dimensional Limit”, Tokyo J. of Math., 30(1), pp. 57-72.

[76] Hmidi T. and Keraani S., (2007), "On the global well-posedness of the two-dimensional Boussinesq system with a zero diffusivity”, Adv. Diff. Eq., 12(4), pp. 461-480.

[77] Abidi H. and Hmidi T., (2007), "On the global well-posedness for Boussinesq system", J. Diff. Eq., 233(1), pp. 199-220.

[78] Attaoui A., (2009), "On the coupling between a Navier-Stokes equation and a total energy equation in spatial dimension N=3", J. Diff. Eq., 246(12), pp. 4591-4617.

[79] Blanchard, D., Bruyere, N., and Guibé, O. (2013), Existence and uniqueness of the solution of a Boussinesq system with nonlinear dissipation, Communications on Pure and Applied analysis, 12(5), 2213-2227. 